\title{
Risk factors of recurrence following common bile duct exploration for choledocholithiasis
}

\author{
Hyun Hwa Choi', Seog-Ki Min ${ }^{2,3}$, Hyeon Kook Lee ${ }^{2,3}$, Huisong Lee',3 \\ 'Department of Surgery, Ewha Womans University Mokdong Hospital, Seoul, Korea \\ ${ }^{2}$ Department of Surgery, Ewha Womans University Seoul Hospital, Seoul, Korea \\ ${ }^{3}$ Department of Surgery, Ewha Womans University College of Medicine, Seoul, Korea
}

Purpose: The purpose of this study was to investigate the recurrence factors of choledocholithiasis after common bile duct (CBD) exploration.

Methods: From January 2000 to December 2018, we retrospectively reviewed 253 patients who underwent CBD exploration surgery. We excluded 100 cases who had residual stone, combined major surgery, or follow-up loss after surgery. Total of 153 patients were included, and we investigated the recurrence factors of choledocholithiasis. Various variables such as patients' demographics, gallstones, preoperative endoscopic treatment, and laboratory data were analyzed to find factors related to recurrent choledocholithiasis.

Results: The median follow-up period was 20.6 months (range 4.7-219 months), and 27 patients (17.6\%) had experienced recurrent choledocholithiasis. Univariate analysis showed that the following variables were associated with recurrence of choledocholithiasis; preoperative leukocytosis (white blood cell $\geq 11,000 / \mu \mathrm{L}$ ), open procedure, $\mathrm{T}$ tube insertion, long hospital duration, and long operation time. Logistic regression multivariate analysis identified preoperative leukocytosis (odds ratio [OR], 3.43; 95\% confidence interval [CI], 1.21-9.73; $p=0.021$ ), open procedure (OR, 5.54; 95\% CI, 4.73-6.35; $p=0.037)$, and T-tube insertion (OR, $2.82 ; 95 \% \mathrm{CI}, 1.04-7.65 ; p=0.042$ ) as independent predictors of recurrent choledocholithiasis.

Conclusion: Because of delayed recurrence of choledocholithiasis, it is recommended to continue follow-up of patients after CBD exploration surgery. Laparoscopic surgery was observed to be associated with a reduction in recurrence. The preoperative leukocytosis and clinical conditions in which open surgery is performed could be associated with recurrence of choledocholithiasis. However, further study is necessary to validate the result.

Keywords: Common bile duct, Gall stones, Recurrence, Risk factors, Laparoscopic cholecystectomy

This is an Open Access article distributed under the terms of the Creative Commons Attribution Non-Commercial License (http:// creativecommons.org/licenses/by-nc/4.0/) which permits unrestricted non-commercial use, distribution, and reproduction in any medium, provided the original work is properly cited.
Received September 10, 2020

Revised 1st October 22, 2020

2nd November 4, 2020

3rd November 6, 2020

Accepted November 6, 2020

Corresponding author

Huisong Lee

Department of Surgery, Ewha

Womans University Mokdong

Hospital, 1071 Anyangcheon-ro,

Yangcheon-gu, Seoul 07985, Korea

Tel: +82-2-2650-5694

Fax: +82-2-2644-7984

E-mail: denebhs@gmail.com

ORCID:

https://orcid.org/0000-0002-3565-6064
Copyright @ 2021 The Journal of Minimally Invasive Surgery. All rights reserved.

\section{INTRODUCTION}

Unlike gallstone, common bile duct (CBD) stone should be removed even in asymptomatic patients because it commonly causes serious biliary complications, such as acute cholangitis or biliary sepsis. An endoscopic retrograde cholangiopancreatog- raphy (ERCP) with endoscopic sphincterotomy (EST) before or after cholecystectomy as a two-stage procedure has been widely accepted as an effective and minimally invasive treatment for $\mathrm{CBD}$ stone. Another approach for the management of choledocholithiasis is a one-stage procedure involving laparoscopic CBD exploration (LCBDE) and laparoscopic cholecystectomy (LC) [1-3]. 
To date, the best treatment options for choledocholithiasis remain unclear. Recent meta-analyses published by Singh and Kilambi [4] and Schacher et al. [5] showed that there was no significant difference between ERCP and LCBDE regarding mortality and complications. However, the length of hospital stay was shorter with surgical treatment than it was with ERCP according to Singh and Kilambi [4]. Furthermore, data from other metaanalyses by Li et al. [3] found surgery to be superior to ERCP since there was greater bile duct clearance and shorter hospital stay in the surgical group with no statistical difference regarding mortality and total postoperative morbidity [6].

The postoperative complication rates after CBD stone treatment is $8 \%$ to $10 \%$; including stone recurrence, pancreatitis, and especially iatrogenic injury to the sphincter of Oddi. The LCBDE with primary duct closure or T-tube drainage are widely accepted as the best treatment option for CBD stone $[7,8]$.

In previous studies, the recurrence rate of choledocholithiasis was $7 \%$ after the endoscopic treatment alone, $18.5 \%$ after the two-stage procedure, and $14.1 \%$ after the one-stage procedure. However, a prospective study by Ding et al. [9] showed that CBD stone recurred in $10 \%$ of treated choledocholithiasis patients and that the recurrence rate of CBD stone in patients treated with the two-stage procedure was five times higher than those treated with the one-stage procedure.

Studies for the recurrence rate of CBD stone after choledocholithiasis investigated the advantages of the one-stage and two-stage procedure have been actively discussed, but there is only few studies of recurrence factor analysis. In the two-stage procedure, CBD stone number, bile duct angulation, and EST are discussed as factors related to CBD stone recurrence [10-12]. In a multicenter study by Park [13], stone size, CBD diameter, and history of LC were risk factors for CBD stone recurrence after LCBDE. On the other hand, Parra-Membrives et al. [14] showed that age is the only independent risk factor associated with choledocholithiasis recurrence following LCBDE.

Research regarding the probability of $\mathrm{CBD}$ stone recurrence according to the time of observation after LCBDE and the study of recurrence factors remains controversial. If recurrence can be predicted at a specific point in time, it will be easier to determine individual observation and treatment policies based on a patient's condition. The purpose of this study was to investigate factors that could predict the recurrence of choledocholithiasis after CBDE.

\section{MATERIALS AND METHODS}

\section{Patients}

We reviewed computerized medical records and images as follows; abdominal ultrasound, abdominal computed tomography
(CT), magnetic resonance imaging (MRI) cholangiography, or echo-endoscopy, from January 2000 to December 2018. Choledocholithiasis was defined as a case where CBD stone was identified in the medical records and the above images. The follow-up period was from the date CBDE was performed to the date of the hospital visit for recurred CBD stone group and December 2018 for the non-recurred group. The median follow-up period was 20.6 months (range, 4.7-219.0 months).

Patients who diagnosed choledocholithiasis and underwent CBDE from January 2000 to January 2018 were included in this study. Abdominal images was performed on patients who revisited our hospital for follow-up observation, and only patients whose CBD stone was confirmed in follow-up abdominal ultrasound, abdominal CT and MRI cholangiography were included in recurrence group. CBDE included both laparoscopic CBDE and open $\mathrm{CBDE}$, and repair included laparoscopic primary repair, open repair, and T-tube insertion.

We deliberately chose to exclude patients that had follow-up periods of less than 3 months cause this short period was thought to be insufficient to confirm the recurrence of $\mathrm{CBD}$ stone. And cases where CBD stone was observed within 3 months after the operation were excluded as the operation was assumed to be incomplete. Cases in which CBDE was performed concurrently with major surgery such as hepatectomy, hepaticojejunostomy and choledochojejunostomy were also excluded, because it was judged that such major surgery could be a confounding factor in study that CBDE was performed alone.

\section{Surgical procedure}

Four trocars were used for LCBDE. A 10-mm trocar was initially inserted into infraumbilical area with an open cut-down technique. Another $10-\mathrm{mm}$ port was inserted inferior to the xyphoid process under camera-visualization, to the right of the falciform ligament, and two 5-mm ports were placed inferior to the right subcostal margin in the anterior axillary line and in the midclavicular line. After Calot's triangle was dissected, the cystic artery, cystic duct, and CBD were exposed. The cystic artery was clipped and transected. Then the cystic duct was milked towards the gall bladder (GB) to oust any cystic duct stone into the GB, clipped very close the GB and transected. A perpendicular ductomy was performed for about $5 \mathrm{~mm}$ on the anterior surface of the exposed CBD, distal to the cystic-CBD junction. The choledochoscope was inserted through this ductomy for CBD exploration and stone extraction. The CBD could be washed away with $30 \mathrm{~mL}$ of saline via the catheter and small stones may be swept out this way. The choledochoscope was also ascended upward to probe the common hepatic duct and intrahepatic ducts. Stones in the common hepatic duct and intrahepatic ducts were removed with a Cook basket. After the stones were completely removed, LC 
was performed. The ductomy was managed with T-tube insertion or primarily repaired with vicryl 5-0 continuous suture.

\section{Evaluated variables}

Patienst's baseline characteristics such as age, sex, height, weight, body mass index (BMI), body surface area (BSA), laboratory data (white blood cells [WBC] count, aspartate transaminase [AST], alanine transaminase [ALT], alkaline phosphatase [ALP], total bilirubin, direct bilirubin, erythrocyte sedimentation rate [ESR], C-reactive protein [CRP]), GB stone, number of GB stones, number of CBD stones, CBD diameter, operation history, preoperative ERCP, preoperative EST and previous cholecystectomy were collected before operation. The degree to which the CBD was dilated before ERCP or operation was confirmed by images, such as MRI cholangiography or abdominal pelvic CT. Demographic data also included laparoscopic procedure, and T-tube insertion. After operation, day to start soft diet, hospital duration, operation time, and red blood cell (RBC) transfusion were checked.

\section{Definition of leukocytosis}

Leukocytosis, often defined as an elevated WBC count greater than $11,000 / \mu \mathrm{L}$ in nonpregnant adults, is a relatively common finding with a wide differential [15].

\section{Statistic analysis}

Data were analyzed using Microsoft Excel 2007 (Microsoft, Redmond, WA, USA). For statistical analysis, Student $t$ test was used for nonparametric data, two-tailed chi-square test or Fisher test was used to compare differences in frequencies, and multivariate analysis was performed for all variables using a logistic regression test. The Kaplan-Meier method was used to estimate the recurrence-free survival (RFS). All statistics were processed in $\mathrm{R}$ version 2.1 (The R Project for Statistical Computing, Vienna, Austria; http://www.r-project.org) and results were considered statistically significant when $p$ value $<0.05$.

\section{RESULTS}

\section{Patients' characteristics}

Among 253 patients underwent LCBDE and CBDE between 2000 and 2018, 153 were included for this study. Eighty-two patients whose follow-ups were less than 3 months and eight patients with residual CBD stone detectected within 3 months were excluded from this study. In addition, 20 patients were excluded because their CBDE was performed along with following major surgeries; 10 choledochojejunostomy (10 patients), hepaticojejunostomy (one), hepatectomy (six), and gastrectomy (three).

There were no significant differences between the nonrecurred and recurred groups in terms of age, sex, height, weight, BMI, and BSA. No significant differences were also observed in ALT, total bilirubin, direct bilirubin, ESR, CRP, multiple CBD stones, $\mathrm{CBD}$ diameter, GB stone, multiple GB stones, operation history, preoperative ERCP, preoperative EST, preoperative cholecystectomy, soft diet starting date, and RBC transfusion.

WBC and AST count showed significant differences between the non-recurred and recurred group. The median WBC count of non-recurred group was $7,400 / \mu \mathrm{L}$ (range, $2,930-25,400 / \mu \mathrm{L}$ ) while that of recurred group was $8,066 / \mu \mathrm{L}$ (range, 3,100-60,880/ $\mu \mathrm{L})(p<0.001)$. The median AST count of non-recurred group was $88.2 \mathrm{IU} / \mathrm{L}$ (range, 11-1,499 IU/L), while that of recurred group was 72 IU/L (range, 17-498 IU/L) ( $p=0.031$ ). Laparoscopic procedure was significantly higher in non-recurred group (96.0\%) than in recurred group $(85.2 \%)$ ( $p=0.03)$. However, T-tube insertion was significantly lower in non-recurred group (16.6\%) than in recurred group (37.0\%) $(p=0.017)$.

Hospital duration and operation time were also shown significant differences. The average hospital duration was 7.7 days (range, 3-33 days) in non-recurred group, while that of recurred group was 10.3 days (range, $4-34$ days) ( $p=0.013$ ). The average operation time of non-recurred group was 152 minutes (range, 75-360 minutes), while that of recurred group was 175 minutes (range, 135-305 minutes) $(p=0.024)$ (Table 1).

\section{Recurrence factor analysis}

With 27 CBD stone-recurred patients (17.6\%), a series of logistic regression analyses was conducted to analyze the recurrence of choledocholithiasis after operation. Results from the univariate analysis showed that preoperative leukocytosis (WBC $\geq 11,000 /$ $\mu \mathrm{L})$, open procedure, T-tube insertion, long hospital duration $(\geq 21$ days) and long operation time ( $\geq 240$ minutes) were significantly associated with CBD stone recurrence. However, in the multivariate analysis, preoperative leukocytosis, open procedure, and T-tube insertion factors showed a significant association with recurrence when the last parameters were corrected. The risk of CBD stone recurrence was 3.43 times higher with leukocytosis than with normal WBC level (odds ratio [OR], 3.43; 95\% confidence interval [CI], 1.21-9.73; $p=0.021$ ). Open procedure was observed as a factor that raised the recurrence risk. CBD stone recurrence was 5.54 times higher in the case of open procedure than in laparoscopic procedure (OR, 5.54; 95\% CI, 4.73-6.35; $p=$ 0.037). The recurrence risk was also 2.82 higher when $\mathrm{T}$ tube was inserted than not inserted (OR, 2.82; 95\% CI, 1.04-7.65; $p=0.042$ ).

Patients' sex, age, height, weight, BMI, BSA, preoperative laboratory data (AST, ALT, ALP, total bilirubin, direct bilirubin, EST, and CRP), GB stone, number of GB stones, number of CBD 
Table 1. Characteristics of patients who underwent CBD exploration $(n=153)$

\begin{tabular}{|c|c|c|c|}
\hline Characteristic & Non-recurred group & Recurred group & $p$ value ${ }^{a}$ \\
\hline No. of patients & 126 & 27 & \\
\hline Age $(y r)$ & $67.4 \pm 14.6$ & $66.6 \pm 14.1$ & 0.563 \\
\hline Male sex & $66(52.4)$ & $11(40.7)$ & 0.090 \\
\hline Height (cm) & $160 \pm 9.4$ & $160.3 \pm 10.4$ & 0.925 \\
\hline Weight (kg) & $60.6 \pm 12.6$ & $57.5 \pm 11.1$ & 0.233 \\
\hline Body mass index $\left(\mathrm{kg} / \mathrm{m}^{2}\right)$ & $23.5 \pm 3.4$ & $22.2 \pm 2.6$ & 0.070 \\
\hline Body surface area & $1.6 \pm 0.2$ & $1.6 \pm 0.2$ & 0.350 \\
\hline WBC (/ML) & $7,400(2,930-25,400)$ & $8,066(3,100-60,880)$ & $<0.001^{*}$ \\
\hline Leukocytosis (WBC $\geq 11,000 / \mu L)$ & $16(12.7)$ & $8(29.6)$ & $0.028^{*}$ \\
\hline AST (IU/L) & $88.2(11-1,499)$ & $72.0(17-498)$ & $0.031^{*}$ \\
\hline ALT (IU/L) & $125(9-891)$ & $171(9-427)$ & 0.274 \\
\hline ALP (IU/L) & $459(110-2,015)$ & $408(174-1,252)$ & 0.168 \\
\hline Total bilirubin (mg/dL) & $1.8(0.2-32.9)$ & $3.1(0.4-9.8)$ & 0.412 \\
\hline Direct bilirubin (mg/dL) & $1.0(0.1-19.8)$ & $2.8(0.1-8.4)$ & 0.637 \\
\hline ESR (mg/dL) & $31(2-91)$ & $34(2-119)$ & 0.615 \\
\hline $\mathrm{CRP}(\mathrm{mg} / \mathrm{dL})$ & $4.2(0.1-29.6)$ & $6.7(3.7-9.8)$ & 0.500 \\
\hline Multiple CBD stones & $77(61.6)$ & $16(59.3)$ & 0.858 \\
\hline CBD diameter (mm) & $12.4 \pm 4.0$ & $12.2 \pm 4.1$ & 0.333 \\
\hline GB stone & $84(66.6)$ & $13(48.1)$ & 0.070 \\
\hline Multiple GB stones & $71(56.3)$ & $10(37.0)$ & 0.068 \\
\hline Opereation history & $48(38.1)$ & $11(40.7)$ & 0.688 \\
\hline Preoperative ERCP & $35(27.7)$ & $3(11.1)$ & 0.069 \\
\hline Preoperative EST & $22(17.5)$ & $3(11.1)$ & 0.418 \\
\hline Previous cholecystectomy & $7(5.6)$ & $0(0)$ & 0.210 \\
\hline Laparoscopic procedure & $121(96.0)$ & $23(85.2)$ & $0.030^{*}$ \\
\hline T-tube insertion & $21(16.7)$ & $10(37.0)$ & $0.017^{*}$ \\
\hline Hospital duration (day) & 7.7 (3-33) & $10.3(4-34)$ & $0.013^{*}$ \\
\hline Operation time (min) & $152(75-360)$ & 175 (135-305) & $0.024^{*}$ \\
\hline RBC transfusion & $4(3.2)$ & $2(7.4)$ & 0.304 \\
\hline
\end{tabular}

Values are presented as number only, mean \pm standard deviation, number (\%), or median (range).

CBD, common bile duct; WBC, white blood cell; AST, aspartate aminotransferase; ALT, alanine aminotransferase; ALP, alkaline phosphatase; ESR, erythrocyte sedimentation rate; CRP, C-reactive protein; GB, gall bladder; ERCP, endoscopic retrograde cholangiopancreatography; EST, endoscopic sphincterotomy; RBC, red blood cell.

${ }^{a}$ Chi-square test, Fisher exact test, or Mann-Whitney U test.

${ }^{*} p<0.05$.

stones, CBD diameter, operation history, preoperative ERCP, preoperative EST, previous cholecystectomy, soft diet starting date, and $\mathrm{RBC}$ transfusion were not related to recurrence of $\mathrm{CBD}$ stone (Table 2).

\section{Recurrence-free survival analysis}

RFS for all patients with CBDE were conducted using the Kaplan-Meier method. The 1-, 3-, and 5-year recurrence rates were estimated 5.2\% (95\% CI, 3.3-7.1), 24.2\% (95\% CI, 19.4-29.0), 
Table 2. Risk factors of CBD stone recurrence after CBD exploration

\begin{tabular}{|c|c|c|c|c|}
\hline \multirow{2}{*}{ Variable } & \multicolumn{2}{|c|}{ Univariate analysis } & \multicolumn{2}{|c|}{ Multivariate analysis ${ }^{\text {a) }}$} \\
\hline & $\mathrm{OR}(95 \% \mathrm{Cl})$ & $p$ value & $\mathrm{OR}(95 \% \mathrm{Cl})^{\mathrm{b})}$ & $p$ value \\
\hline Age (yr) & $0.99(0.96-1.02)$ & 0.523 & & \\
\hline Male sex & $0.77(0.33-1.79)$ & 0.542 & & \\
\hline Body mass index (kg/m²) & $0.88(0.77-1.01)$ & 0.072 & & \\
\hline Leukocytosis (WBC $\geq 11,000 / \mu L)$ & $2.89(1.09-7.70)$ & $0.033^{*}$ & $3.43(1.21-9.73)$ & $0.021^{*}$ \\
\hline Previous EST & $0.59(0.16-2.14)$ & 0.422 & & \\
\hline Open procedure & $4.21(3.51-4.91)$ & $0.042^{*}$ & $5.54(4.73-6.35)$ & $0.037^{*}$ \\
\hline T-tube insertion & $2.90(1.18-7.31)$ & $0.020^{*}$ & $2.82(1.04-7.65)$ & $0.042^{*}$ \\
\hline Long hospital duration ( $\geq 21$ days) & $3.86(1.24-13.28)$ & $0.032^{*}$ & $1.65(0.38-7.15)$ & 0.503 \\
\hline Long operation time ( $\geq 240 \mathrm{~min}$ ) & $2.95(0.90-9.66)$ & $0.053^{*}$ & $2.05(0.55-7.60)$ & 0.284 \\
\hline
\end{tabular}

$\mathrm{CBD}$, common bile duct; OR, odds ratio; Cl, confidence interval; WBC, white blood cell; EST, endoscopic sphincterotomy.

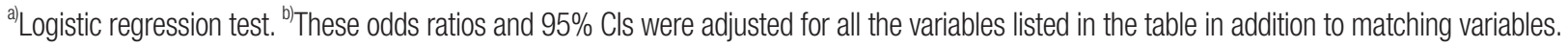
${ }^{*} p<0.05$.

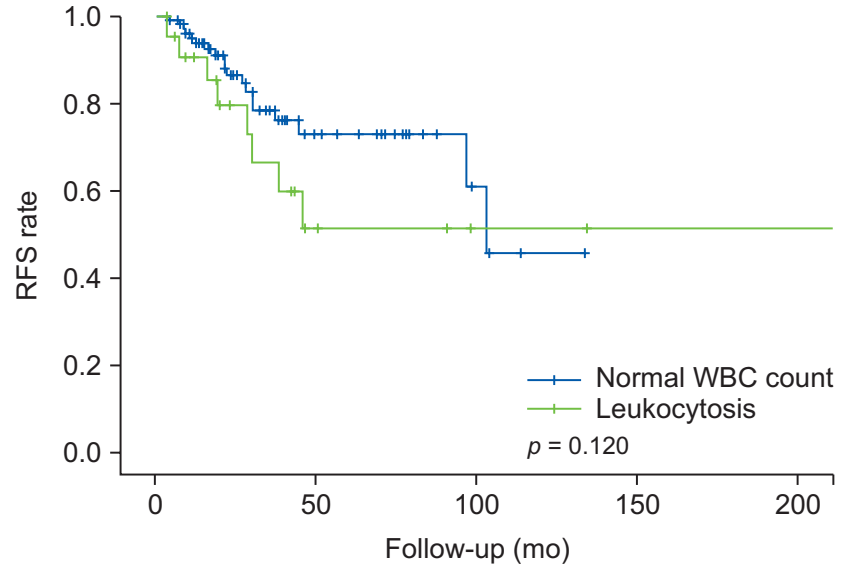

Fig. 1. Comparison of recurrence-free survival (RFS) rate between normal white blood cell (WBC) count $(<11,000 / \mu \mathrm{L})$ and leukocytosis (WBC $\geq 11,000 / \mu \mathrm{L})$. No significant difference in RFS rate was found between the two groups.

and 30.6\% (95\% CI, 25.0-36.2), respectively. The RFS between the normal WBC count group (WBC $<11,000 / \mu \mathrm{L}$ ) and leukocytosis group (WBC $\geq 11,000 / \mu \mathrm{L}$ ) were compared and no significant difference was found between the two groups $(p=0.120)$. The 1 -, 3 -, and 5-year recurrence rates of the normal WBC count group were estimated $0.8 \%$ (95\% CI, 0.73-0.87), 3.6\% (95\% CI, 1.8-5.4), and $6.9 \%$ (95\% CI, 4.4-9.4), respectively. In leukocytosis group, the recurrence rates were estimated $20.0 \%$ (95\% CI, 10.9-29.1), $33.3 \%$ (95\% CI, 21.9-44.7), and 48.6\% (95\% CI, 35.5-61.7), respectively (Fig. 1).

The RFS between open CBDE group and laparoscopic CBDE group were also analyzed and the result showed significant dif-

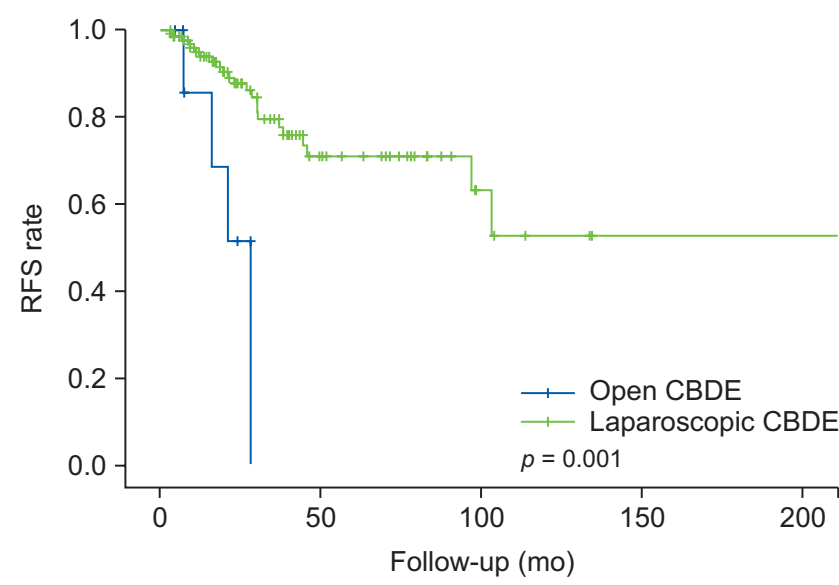

Fig. 2. Comparison of recurrence-free survival (RFS) rate between open common bile duct exploration (CBDE) and laparoscopic CBDE. The result showed significant difference in RFS rate between the two groups.

ference $(p=0.001)$. The 1-, 3-, and 5-year recurrence rates of open CBDE group were estimated 31.4\% (95\% CI, 12.8-50.0), 48.6\% (95\% CI, 28.2-69.0), and unpredictable, respectively; while in laparoscopic CBDE group, they were estimated 6.1\% (95\% CI, 4.1-8.1), 20.4\% (95\% CI, 15.8-25.0), and 29.0\% (95\% CI, 23.2-34.8), respectively (Fig. 2).

\section{DISCUSSION}

CBD stone usually originates in the GB and then they migrate into the CBD. Therefore, cholecystectomy would be helpful in preventing $\mathrm{CBD}$ stone recurrence. However, the recurrence of $\mathrm{CBD}$ stone is still observed in some patients following cholecys- 
tectomy. There have been several studies about the prevalence and the risk factors for CBD stone recurrence after EST, but little data is available on CBD stone recurrence after cholecystectomy. Although LCBDE is considered a safe and effective procedure for patients with ERCP failure, it is also an effective treatment for CBD stone suspected at cholecystectomy. However, CBD stone may still recur even after LCBDE [1,16-18].

Recurrent choledocholithiasis is the most common side effect observed in the follow-up process after successful CBD stone removal and occurs with a frequency of $4 \%$ to $24 \%$ usually within 6 years $[19,20]$. Primary CBD stone can recur after cholecystectomy because stones are formed in the bile duct due to bile stasis. Many studies have reported that CBD stone is associated with bile duct stricture, papillary stenosis, periampullary diverticulum, reflux of duodenal contents into the bile duct, parasites or foreign bodies within the bile duct, or other factors predisposing to stasis and encouraging bacterial overgrowth. But the risk factors are still not clear [10].

Several studies have shown that CBD stone size, stone numbers, CBD dilatation, sharp angulation, and history of LC or ERCP with EST are related to the recurrence of CBD stone [21-23]. Our study showed that risk factors for $\mathrm{CBD}$ stone recurrence after CBDE were preoperative leukocytosis (WBC $\geq 11,000 / \mu \mathrm{L}$ ), laparoscopic procedure, and T-tube insertion. This finding differs from the results of previous studies that identified risk factors for $\mathrm{CBD}$ stone recurrence after ERCP and cholecystectomy. Leukocytosis, where the WBC count is $11,000 / \mu \mathrm{L}$ or higher, was a unique factor that has not been reported as a significant risk factor of recurrence in previous studies. Probably, there may be question about whether there are other underlying diseases of the patient that can be cause leukocytosis. The underlying disease and preoperative condition of the patients were investigated. The frequency of the underlying disease is as follows: diabetes, 31 patients; hypertension, 53; asthma, 6; cerebrovascular accident, 7; angina, 3; hepatitis $B$ virus (HBV) infection, 6; hepatatis $C$ virus (HCV), 1; pneumonia, 3; and sepsis, 2. Twelve patients, including $\mathrm{HBV}, \mathrm{HCV}$, pneumonia, and sepsis, can be direct cause of leukocytosis, which is very few accounting for $7.8 \%$ of all patients.

There are many studies that have shown that inflammation has a significant effect on CBD stone formation. During choledocholithiasis inflammation, oxidative stress promotes mitochondrial dysfunction in hepatocytes, which mainly leads to necrosis. Malondialdehyde, a product of oxidative stress, is extremely cytotoxic and causes damage to cell membranes and intracellular macromolecules. In addition, the presence of endocrine cells (ECs) may be related to bile flow disruption and calculi formation. EC hyperplasia may be related to prolonged inflammation as chronic cholecystitis and all EC secreted hormones can support the pathologic process in the choledochus (i.e., inflammation, increased mucus secretion, fibrosis, muscle contraction, etc.).
Various ECs (similar to those in the duodenum) that occupy the lower region of the large bile duct and their hormones act on the physiology (motility and secretion) and pathology (inflammation and fibrosis) of the biliary tree in the area $[16,24]$. Our study is the first report of preoperative leukocytosis associated with CBD stone recurrence. It is speculated that leukocytosis prior to choledocholithiasis management can be a predictor of recurrence as it affects a patient's inflammation level, which reflects the pathophysiological severity of the CBD condition.

In our RFS analysis, the 1-, 3-, and 5-year recurrence rates of all patients with CBDE were 5.2\%, 24.2\%, and 30.6\%, respectively. After dividing patients into normal WBC count group and leukocytosis group, the 1-, 3-, and 5-year recurrence rates of the former group were $0.8 \%, 3.6 \%$, and $6.9 \%$, respectively; while in the latter group, the rates were $20.0 \%, 33.3 \%$, and $48.6 \%$, respectively. Recurrence of CBD stone occurred in almost half of patients with preoperative leukocytosis and these patients had a 7-fold increase in the probability of recurrence after 5 years than patients with a preoperative normal WBC count. This result suggests that patients with preoperative leukocytosis require continuous follow-ups for more than 5 years to monitor CBD stone recurrence. Moreover, the 1-, 3-, and 5-year recurrence rates of open CBDE group were estimated $31.4 \%, 48.6 \%$, and unpredictable, respectively; while in laparoscopic CBDE group, the rates were $6.1 \%$, $20.4 \%$, and 29.0\%, respectively. Open CBDE had 2.38 times higher probability of $\mathrm{CBD}$ stone recurrence than laparoscopic $\mathrm{CBDE}$ after 3 years. This suggests that choosing laparoscopy is advantageous for the purpose of preventing $\mathrm{CBD}$ stone recurrence. The reason that laparoscopic procedure lowered the recurrence of CBD stone was thought to be that the CBD inflammatory condition of patients with open CBDE might be worse than patients with laparoscopic CBDE. In other words, it can be explained that if the inflammatory state of CBD is much more serious, open procedure can be chosen instead of laparoscopy.

Comments on T-tube insertion are controversial. Ozcan et al. [25] showed that percutaneous CBD expulsion into the duodenum through the T-tube tract is a non-traumatic, effective, and safe method for the treatment of residual CBD stone in patients who had a cholecystectomy and T-tube insertion. Contrariwise, El-Geidie [26] demonstrated in a randomized study that LCBDE with primary closure without external drainage after laparoscopic choledochotomy is feasible, safe, and cost-effective. In our multivariate analysis, CBD stone recurrence was 2.82 times higher when T-tube was inserted than when T-tube was not inserted (OR, 2.82; 95\% CI, 1.04-7.65; $p=0.042$ ). T-tube insertion was performed when bile duct wall was dirty or if the surgeon determined that there was a possibility of CBD stone recurrence during operation, which may cause selection bias.

In a retrospective study by Choi et al. [21], patients over the age of 65 years was a risk factor of CBD stone recurrence after LC. In 
addition, Parra-Membrives et al. [14] showed that age is the only independent risk factor associated to choledocholithiasis recurrence following LCBDE [14,21]. In our study, the mean age of patients was $68.0 \pm 14.2$ years. But there was no evidence that old age is a risk factor for CBD stone recurrence in our data.

The definitions of residual and recurrent CBD stone are somewhat different depending on the reporters, but generally residual CBD stone is not found during surgery but found within 3 months after surgery. In our study, the median follow-up period was 20.6 months (range, 4.7-219.0 months) after follow-up periods of less than 3 months and cases where CBD stone was observed within 3 months after CBDE were excluded. It was thought that $\mathrm{CBD}$ stone assessed as recurrence after operation was reliably excluded residual stone.

This study had three limitations. First, our study is a retrospective study, so it is carrying an inherent bias in data collection. Second, there is a possibility that leukocytosis did not reflect choledocholithiasis but the degree of inflammation of the patient's general condition, such as hepatitis, pneumonia, and sepsis. Third, it is thought that the patients who underwent open procedure were relatively difficult cases. Therefore, the association between laparoscopic procedure and recurrence reduction suggests high possibility of selection bias.

In conclusion, because of delayed recurrence of choledocholithiasis, it is recommended to continue follow-up of patients after CBD exploration surgery. Laparoscopic surgery was observed to be associated with a reduction in recurrence. The preoperative leukocytosis and clinical conditions in which open surgery is performed could be associated with recurrence of $c$ choledocholithiasis. However, further study is necessary to validate the result.

\section{NOTES}

\section{Ethical statements}

This retrospective study was approved by the Institutional Review Board (IRB) of Ewha Womans University Mokdong Hospital (No. 2020-05-028). Written informed consent was waived by the IRB.

\section{Authors' contributions}

Conceptualization: HL

Data curation: HHC

Formal analysis: SKM

Funding acquisition: SKM, HKL

Investigation: SKM, HKL

Methodology: HL

Project administration: $\mathrm{HHC}, \mathrm{HL}$

Visualization: HHC
Writing-original draft: HHC, HL

Writing-review \& editing: All authors

All authors read and approved the final manuscript.

\section{Conflict of interest}

All authors have no conflicts of interest to declare.

\section{ORCID}

Hyun Hwa Choi, https://orcid.org/0000-0003-1476-5410

Seog-Ki Min, https://orcid.org/0000-0001-9894-7603

Hyeon Kook Lee, https://orcid.org/0000-0002-7975-2672

Huisong Lee, https://orcid.org/0000-0002-3565-6064

\section{REFERENCES}

1. Choi K, Amarasena T, Hughes A, et al. Management of bile duct stones at cholecystectomy: an Australian single-centre experience over 2 years. Surg Endosc 2020 Mar 9 [Epub]. https://doi.org/10.1007/ s00464-020-07495-7.

2. Voyles CR, Sanders DL, Hogan R. Common bile duct evaluation in the era of laparoscopic cholecystectomy. 1050 cases later. Ann Surg 1994;219:744-750.

3. Li ZQ, Sun JX, Li B, Dai XQ, Yu AX, Li ZF. Meta-analysis of singlestage versus two-staged management for concomitant gallstones and common bile duct stones. J Minim Access Surg 2020;16:206-214.

4. Singh AN, Kilambi R. Single-stage laparoscopic common bile duct exploration and cholecystectomy versus two-stage endoscopic stone extraction followed by laparoscopic cholecystectomy for patients with gallbladder stones with common bile duct stones: systematic review and meta-analysis of randomized trials with trial sequential analysis. Surg Endosc 2018;32:3763-3776.

5. Schacher FC, Giongo SM, Teixeira FJ, Mattos ÂZ. Endoscopic retrograde cholangiopancreatography versus surgery for choledocholithiasis: a meta-analysis. Ann Hepatol 2019;18:595-600.

6. Gantois D, Goudard Y, Bourgouin S, Pauleau G, de La Villéon B, Balandraud P. One-stage laparoscopic procedure versus two-stage procedure in the management of common bile duct stones in patients aged 75 and more. J Visc Surg 2020;157:99-106.

7. He MY, Zhou XD, Chen H, Zheng P, Zhang FZ, Ren WW. Various approaches of laparoscopic common bile duct exploration plus primary duct closure for choledocholithiasis: a systematic review and meta-analysis. Hepatobiliary Pancreat Dis Int 2018;17:183-191.

8. Shojaiefard A, Esmaeilzadeh M, Ghafouri A, Mehrabi A. Various techniques for the surgical treatment of common bile duct stones: a meta review. Gastroenterol Res Pract 2009;2009:840208.

9. Ding G, Cai W, Qin M. Single-stage vs. two-stage management for concomitant gallstones and common bile duct stones: a prospective randomized trial with long-term follow-up. J Gastrointest Surg 2014; 
18:947-951.

10. Yoo ES, Yoo BM, Kim JH, et al. Evaluation of risk factors for recurrent primary common bile duct stone in patients with cholecystectomy. Scand J Gastroenterol 2018;53:466-470.

11. Ha DW, Song GA, Kim DU, et al. Recurrent common bile duct stone and endoscopic treatment after endoscopic papillary large balloon dilatation with minor endoscopic sphincterotomy. Korean J Gastroenterol 2011;57:352-357.

12. Pan L, Chen M, Ji L, et al. The safety and efficacy of laparoscopic common bile duct exploration combined with cholecystectomy for the management of cholecysto-choledocholithiasis: an up-to-date meta-analysis. Ann Surg 2018;268:247-253

13. Park $\mathrm{CH}$. The management of common bile duct stones. Korean J Gastroenterol 2018;71:260-263.

14. Parra-Membrives P, Martínez-Baena D, Lorente-Herce JM, JiménezRiera G, Sánchez-Gálvez MÁ. Choledocholithiasis recurrence following laparoscopic common bile duct exploration. Cir Esp 2019;97:336342.

15. Riley LK, Rupert J. Evaluation of patients with leukocytosis. Am Fam Physician 2015;92:1004-1011.

16. Cai JS, Qiang S, Bao-Bing Y. Advances of recurrent risk factors and management of choledocholithiasis. Scand J Gastroenterol 2017;52: 34-43.

17. Al-Temimi MH, Kim EG, Chandrasekaran B, et al. Laparoscopic common bile duct exploration versus endoscopic retrograde cholangiopancreatography for choledocholithiasis found at time of laparoscopic cholecystectomy: analysis of a large integrated health care system database. Am J Surg 2017;214:1075-1079.

18. Gomez D, Cabrera LF, Villarreal R, et al. Laparoscopic common bile duct exploration with primary closure after failed endoscopic retrograde cholangiopancreatography without intraoperative cholangiography: a case series from a referral center in Bogota, Colombia. J Laparoendosc Adv Surg Tech A 2020;30:267-272.

19. Prat F, Malak NA, Pelletier G, et al. Biliary symptoms and complications more than 8 years after endoscopic sphincterotomy for choledocholithiasis. Gastroenterology 1996;110:894-899.

20. Keizman D, Shalom MI, Konikoff FM. An angulated common bile duct predisposes to recurrent symptomatic bile duct stones after endoscopic stone extraction. Surg Endosc 2006;20:1594-1599.

21. Choi YS, Do JH, Suh SW, Lee SE, Kang H, Park HJ. Risk factors for the late development of common bile duct stones after laparoscopic cholecystectomy. Surg Endosc 2017;31:4857-4862.

22. Chong CC, Chiu PW, Tan T, et al. Correlation of CBD/CHD angulation with recurrent cholangitis in patients treated with ERCP. Endosc Int Open 2016;4:E62-E67.

23. Baek YH, Kim HJ, Park JH, et al. Risk factors for recurrent bile duct stones after endoscopic clearance of common bile duct stones. Korean J Gastroenterol 2009;54:36-41.

24. Gulubov MV, Hadjipetkov P, Sivrev D, Ilieva G. Endocrine cells in the human common bile duct in patients with obstructive jaundice. Hepatogastroenterology 2012;59:26-30.

25. Ozcan N, Kahriman G, Karabiyik O, Donmez H, Emek E. Percutaneous management of residual bile duct stones through T-tube tract after cholecystectomy: a retrospective analysis of 89 patients. Diagn Interv Imaging 2017;98:149-153.

26. El-Geidie AA. Is the use of T-tube necessary after laparoscopic choledochotomy? J Gastrointest Surg 2010;14:844-848. 